\title{
Editorial
}

\section{Is There Room for Paradox in CAM?}

\author{
Edwin L. Cooper
}

\begin{abstract}
Laboratory of Comparative Neuroimmunology, Department of Neurobiology, David Geffen School of Medicine at UCLA, Box 951763, 63-230 CHS, Los Angeles California 90095-1763, USA
\end{abstract}

The field of CAM is fluid, ever changing and ever approaching the essence of modern western medicine using the evidence based approach (1). However, since some ancient practices continue without scientific evidence, there are still international perceptions that CAM is mired in folk remedies. This situation is most pertinent to TCM as expressed in an editorial about the debate in China by Mark Magnier, Times Staff Writer of The Los Angeles Times, Monday, January 8, 2007 entitled: Scalpel vs. Herb in China.

The debate in China concerns ancient remedies and their value in contrast to the universally prevalent and relatively young model of Western Medicine. Members of international populations without vital traditional medicine have found that these ancient remedies possess a cornucopia of CAM options when treating various afflictions-mostly chronic. In particular, those with prognoses that escape western medicine choose a treatment regimen of CAM alone or a combination of CAM linked to western medical practice. In China, on the other hand, traditional medicine based on thousands of years of history is still practiced far from the frenzy of pharmaceutical geniuses and their seemingly conflicting cures, and has been able to skirt the expense and profit driven motives of western medical doctors and pharmaceutical industries. Yet now, with an increasing presence of western medicine, many Chinese have become aware of the limitations of their ancient practice just as westerners question their own historically younger practice. Here I perceive an apparent paradox, if we view the realities in China, Europe, Australia and North America. According to the commonly perceived

For reprints and correspondence: Edwin L. Cooper, Laboratory of Comparative Neuroimmunology, Department of Neurobiology, David Geffen School Of Medicine at UCLA, Box 951763, 63-230 CHS,

Los Angeles California 90095-1763, USA. Tel: (310) 825-9567;

Fax: (310) 825-2224; E-mail: ecam@mednet.ucla.edu definition of a paradox, we know the following: A paradox is a seemingly absurd or contradictory statement, even if actually well founded. Here is an excerpt of what Mark Magnier wrote that set off my examination of this apparent paradox.

'A relatively obscure professor at a regional university kicked off the controversy in October with an online petition calling for traditional medicine to be stripped from the Chinese Constitution. It has protected status here that, at least in theory, guarantees it equal footing with its Western counterpart. Professor Zhang Gongyao and fellow critics have blasted Chinese medicine as an often ineffective, even dangerous derivative of witchcraft that relies on untested concoctions and obscure ingredients to trick patients, then employs a host of excuses if the treatment doesn't work. For adherents of the 3,000-year-old system, this borders on heresy. The Health Ministry labeled Zhang's ideas "ignorant of history," and traditionalists have called the skeptics traitors bent on "murdering" Chinese culture. Ironically, the firestorm dovetails with a growing embrace of Chinese Medicine abroad as an antidote to the perceived soulless, moneyobsesses nature of Western healthcare. On a trip to China in mid-December, U.S. Health and Human Services Secretary Mike Leavitt said that the two countries planned to trade lessons on how to integrate Western and Chinese medicine. "It's an area of interest for China and the U.S.," he said. Many Australians, Europeans and Americans see the limitations of advanced science, said Rey Tiquia, an expert in Chinese traditional medicine based in Australia, even as more Chinese view their traditions as old-fashioned.'

My main point here concerns the apparent lack of recognition of scientific approaches to TCM being performed in China and internationally. For example, the National Center for Complementary and Alternative Medicine (NCCAM) in the United States actually funds 
highly meritorious peer reviewed projects that promise to understand through evidence based approaches untested claims that continue to fuel the conceptions and misconceptions of these ancient practices. Then there are the avenues for informing all of us what actually may occur through well-designed analyses. And this comes about through the rigors of well-designed research projects. The approach may apply in vitro strategies or animal models that can be better for immediate deciphering of what may be occurring in humans without the ethical, logistic and personal concerns of human trials.

From a philosophical viewpoint and with the added unique advantage of being a veterinarian, Kaphle, et al. (2) gave a very favorable view of research in Taiwan. 'My first visit to a laboratory at National Taiwan University opened my eyes to the possibilities of herbal medicine research, especially in the area of veterinary medicine. It became my ambition to link the knowledge of Traditional Chinese Medicine (TCM) and Ayurveda from the Indian subcontinent and their integration with other systems of medicine, including Western medicine (WM), to achieve the concept of Sustainable Medicine, firstly for animals and then for humans. The Ministry of Economic Affairs (MOEA) has implemented a technology development program to quickly establish the key technologies, and this is a moment of opportunity for Taiwan's traditional herbal medicine industry to upgrade and transform itself.' This is a clear cut example of how animals can become the focus of TCM approaches to disease rather than the exact opposite, i.e. using their parts to cure diseases of humans - a poignant reminder of what the press and popular views attach to TCM!

And by real advocacy of comparison that promises greater understanding; Patwarddhan et al. (3) offer the following. 'Ayurveda, the traditional Indian medicine (TIM) and traditional Chinese medicine (TCM) remain the most ancient yet living traditions. Increased global interest in traditional medicine has spawned efforts to monitor and regulate herbal drugs and traditional medicine. China has been successful in promoting its therapies with more research and a science-based approach, while Ayurveda still needs more extensive scientific research and evidence base. This overview of basic principles and commonalities of TIM and TCM discusses the key determinants of success that these great traditions need to address to compete in global markets.'

Tan et al. (4) further elucidate the West's turn to the East for answers: 'The so-called functional somatic syndromes comprise a group of disorders that are primarily symptom-based, multisystemic in presentation and probably involve alterations in mind-brain-body interactions. The emerging neurobiological models of allostasis/allostatic load and of the emotional motor system show striking similarities with concepts used by
Traditional Chinese Medicine (TCM) to understand functional somatic disorders and their underlying pathogenesis. These models incorporate a macroscopic perspective, accounting for the toll of acute and chronic traumas, physical and emotional stressors and the complex interactions between the mind, brain and body. The convergence of these biomedical models with the ancient paradigm of TCM may provide a new insight into scientifically verifiable diagnostic and therapeutic approaches for these common disorders.'

Turning to the purely scientific approach of animal models and what they may offer regarding TCM, the linking of nervous and immune systems can yield much that can be extrapolated for humans. According to Wang et al., (5) 'Distress-mediated tissue oxidative stress [has been] examined as a model of sub-healthy condition defined in traditional Chinese medicine [TCM] theory. Mice were subjected to psychologically stressful conditions by whisker removal. The oxidative tissue injuries observed at $12 \mathrm{~h}$ after the removal of the whiskers were effectively prevented by two traditional Chinese medicine formula: Shengmai San (SMS) and Ling Gui Zhu Gan Tang (LGZGT), when administered for 5 days before the removal of the whiskers. It is worth noting that these results made use of statistical analyses to support the significance of their findings, often not the situation in poorly designed human trials (case reports or anecdotes) at the bottom of the golden pyramid and not exemplary of the pinnacle: the randomized, controlled, double blind analyses that are ideal when humans are used as subjects.

How do peer reviewed journals reach the public and what is the impact? Surely research analyses that utilize humans and real chronic problems often attract the greatest attention. Here I refer to substantial coverage of the work of Michael Irwin on Tai Chi [featured April 17, 2007 in the New York Times and The Times (London), and April 15, 2007 in the Los Angeles Times]. Earlier aspects of this work were published in $e C A M$ (Irwin et al., 2004) and showed that both the incidence and severity of herpes zoster (HZ) or shingles increased markedly with age in association with a decline in varicella zoster virus (VZV)-specific immunity (6). In his review, he discussed recent findings that administration of a relaxation response-based intervention, tai chi chih (TCC), results in improvements in health functioning and immunity to VZV in older adults as compared with a control group. Focus on older adults at increased risk for $\mathrm{HZ}$ and assay of VZV-specific immunity have implications for understanding the impact of behavioral factors and a behavioral intervention on a clinically relevant endpoint and on the response of the immune system to infectious pathogens.

Has my opinion been convincing? Citing an apparently old-fashioned view still held in China and yet moving away from it, in contrast to a booming approach to the system 
in the west seems to be the paradox. In the face of progress toward understanding the mechanisms of TCM and Ayurveda, (TIM), (Kampo of Japan was not mentioned) we have presented examples of views that treat humans and use animal models. These results seem to contradict some of the assertions of the opening paragraphs. In my opinion, the Pacific Ocean is narrowing or are scientists at work on a bridge to span the great divide?

\section{References}

1. Cooper EL. Complementary and alternative medicine, when rigorous, can be science. Evid Based Complement Alternat Med 2004;1:1-4.
2. Kaphle K, Wu LS, Yang NJ, Lin JH. 2006 herbal medicine research in Taiwan. Evid Based Complement Alternat Med 2006;3:149-55.

3. Patwardhan B, Warude D, Pushpangadan P, Bhatt N. Ayurveda and traditional Chinese medicine: a comparative overview. Evid Based Complement Alternat Med 2005;2:465-73.

4. Tan S, Tillisch K, Mayer E. Functional somatic syndromes: emerging biomedical models and traditional Chinese medicine. Evid Based Complement Alternat Med 2004;1:35-40.

5. Wang L, Muxin G, Nishida H, Shirakawa C, Sato S, Konishi T. Psychological stress-induced oxidative stress as a model of subhealthy condition and the effect of TCM. Evid Based Complement Alternat Med (Advance Access published on November 2, 2006, DOI 10.1093/ecam/nel080).

6. Irwin M, Pike J, Oxman M. Shingles immunity and health functioning in the elderly: Tai Chi Chih as a behavioral treatment. Evid Based Complement Alternat Med 2004;1:223-232. 


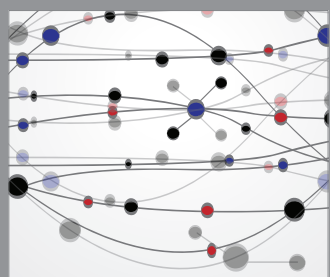

The Scientific World Journal
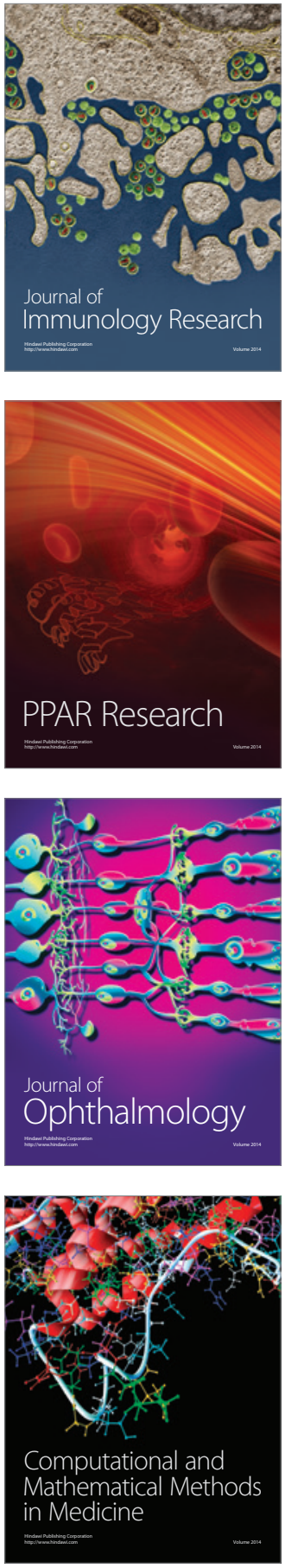

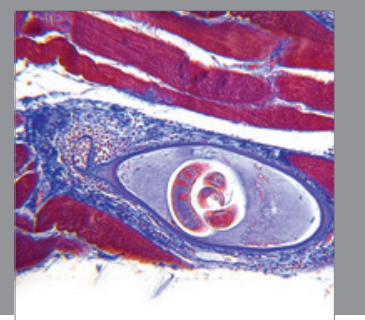

Gastroenterology

Research and Practice
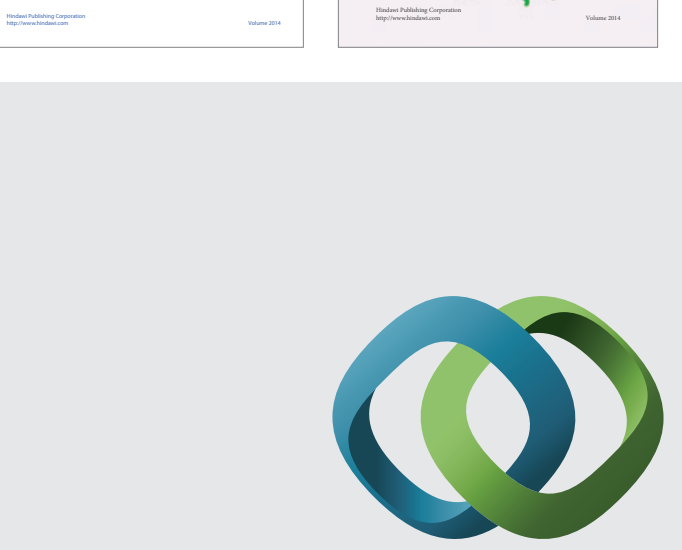

\section{Hindawi}

Submit your manuscripts at

http://www.hindawi.com
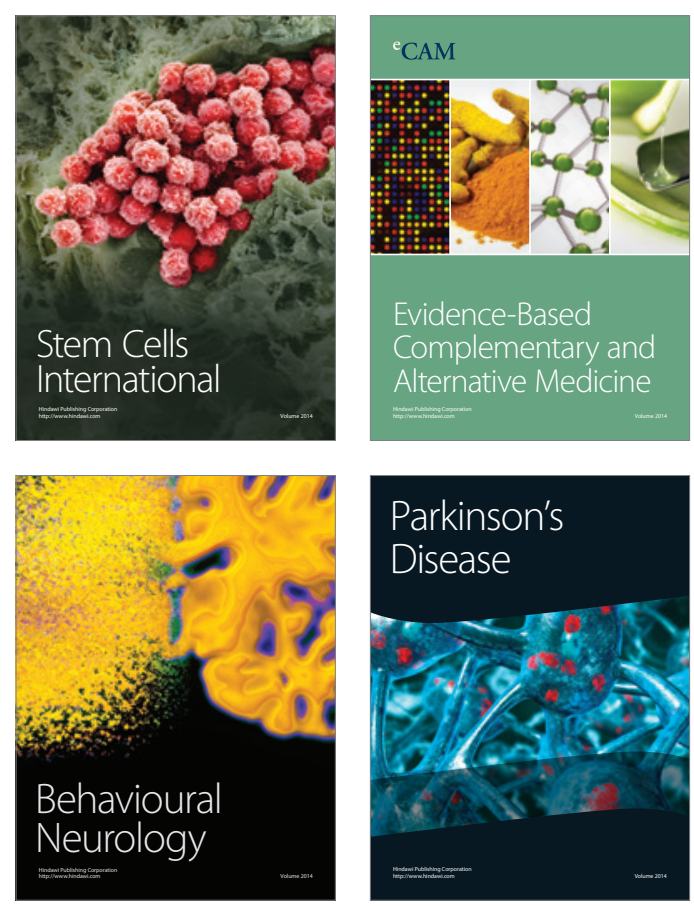

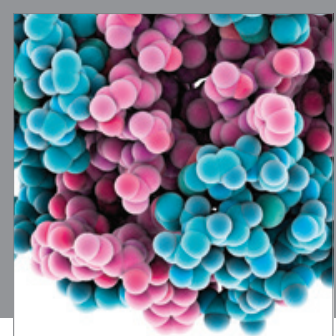

Journal of
Diabetes Research

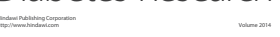

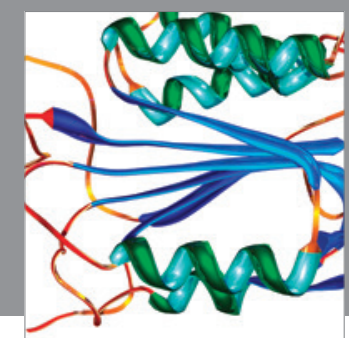

Disease Markers
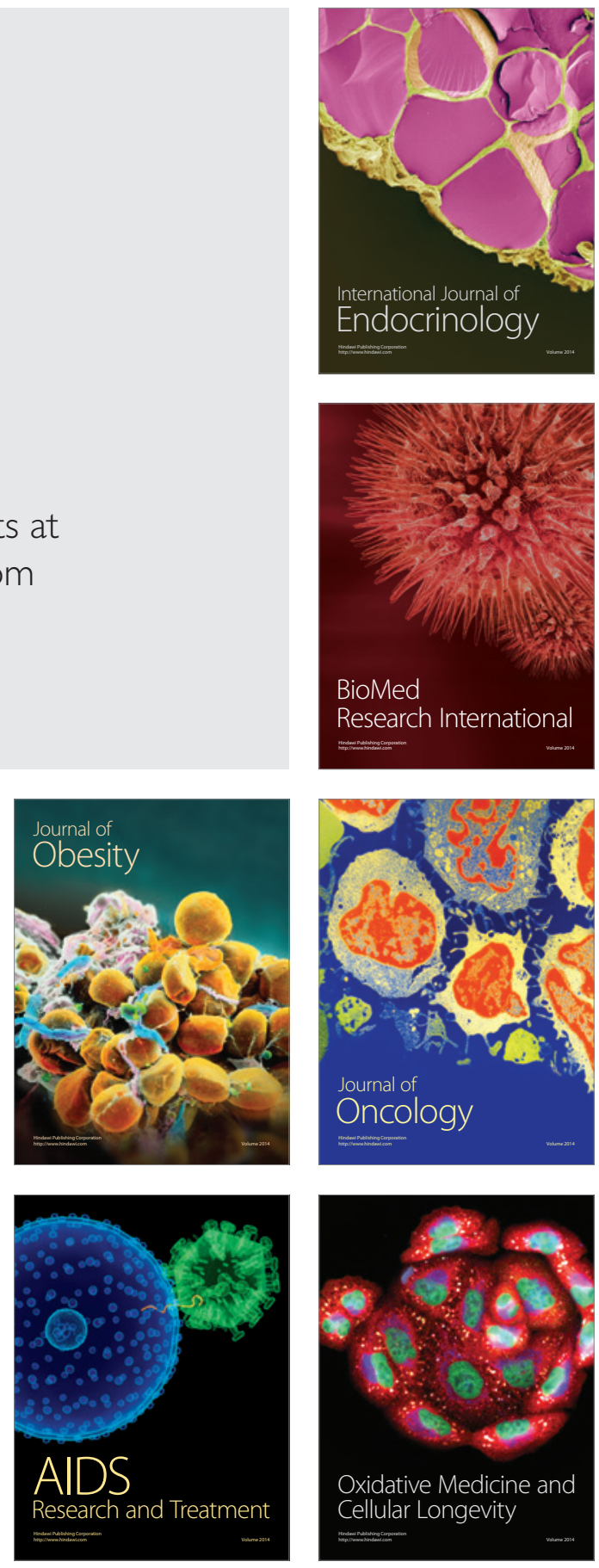